\title{
Supragingival and subgingival microbiota from patients with poor oral hygiene submitted to radiotherapy for head and neck cancer treatment
}

\author{
Elerson Gaetti-Jardim Jr. ${ }^{a, *}$, Ellen Cristina Gaetti Jardim ${ }^{\mathrm{b}}$, Christiane Marie Schweitzer ${ }^{\mathrm{c}}$, \\ Júlio Cesar Leite da Silva ${ }^{\mathrm{b}}$, Murilo Moura Oliveira ${ }^{\mathrm{b}}$, Danilo Chizzolini Masocatto ${ }^{\mathrm{b}}$, \\ Cauê Monteiro dos Santos ${ }^{\mathrm{b}}$ \\ a São Paulo State University (UNESP), School of Dentistry, Department of Pathology and Clinical Propaedeutics, Araçatuba, São Paulo, Brazil \\ b School of Dentistry of Campo Grande, Universidade Federal do Mato Grosso do Sul, Campo Grande, Mato Grosso do Sul, Brazil

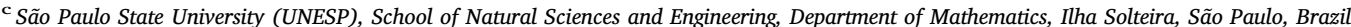

\section{A R T I C L E I N F O}

\section{Keywords:}

Oral cancer

Head and neck radiotherapy

Mucositis

Xerostomia

Microbiology

\begin{abstract}
A B S T R A C T
Objective: This case-control study aimed to evaluate the effects of conventional radiotherapy (RT) on the prevalence and populations of oral microorganisms in head and neck cancer patients who did not receive adequate preventive dental care. It was hypothesized that side effects of radiotherapy could be associated with radiation dose, microbiological aspects, and socioeconomic conditions of the patients.

Design: Twenty-eight dentate patients with head and neck cancer submitted to RT were included in the study. Radiation dose received varied from 4320 to 7020 cGy. Patients with the same demographic and health conditions, but no history of cancer or antineoplastic treatment were used as controls. Clinical examinations were carried out before RT, 15-22 days after starting RT, immediately after and 6 months after RT. Supra and subgingival biofilms were collected and cultivated onto selective and non-selective media. Isolates were identified by biochemical and physiological characteristics. Stimulated and unstimulated salivary flow rate and saliva buffer capacity were also determined.

Results: Mucositis, dermatitis, xerostomia, dysgeusia, dysphagia and candidiasis were common after starting RT and during the treatment period. Xerostomia was followed by a decrease in salivary $\mathrm{pH}$ and buffer capacity, which showed association with the increase of cariogenic cocci and yeast populations, which were also associated with deterioration of hygiene. Candida and family Enterobacteriaceae showed increased prevalence with $\mathrm{RT}$, and were associated with the occurrence of mucositis and xerostomia.

Conclusions: Modifications in oral biofilms of irradiated patients showed association with xerostomia and hygiene conditions, which reinforces the necessity of improving patient compliance to oral health care programs.
\end{abstract}

\section{Introduction}

Radiotherapy (RT) is commonly used to treat head and neck cancer, significantly improving patient survival. However, RT is deeply associated with severe adverse reactions that may affect patient compliance, progress and intensification of the therapeutic regimen due to deterioration of the quality of life. The occurrence and severity of the side effects depend on dosage/frequency of RT, volume and anatomical aspects of irradiated tissues, age, oral and general health conditions of patients, as well as surgical procedures and associated chemotherapy (Minhas, Kashif, Altaf, \& Nagi1, 2016).

Mucositis and xerostomia are the most significant and undesired effects of RT, affecting most irradiated patients, producing extreme discomfort. Xerostomia reflects the action of ionizing irradiation on the salivary glands and may exacerbate the effects of RT on highly proliferative epithelial cells of the oral mucosa, resulting in mucositis (Scarantino et al., 2006).

Reduction of the blood supply occurs in irradiated tissues during RT treatment. Thus, a similar reduction of the redox potential is expected, leading to wound repair delay and reduction of local immunity, which could particularly lead to proliferation of obligate anaerobes and microaerophilic bacteria in the supra or subgingival biofilms (Sonalika, Tayaar, Bhat, Patil, \& Muddapur, 2012). Moreover, radiation-induced xerostomia seems to aggravate the prevalence of non-oral opportunistic pathogens in the oral biofilm of irradiated patients with adequate oral hygiene conditions and previous dental treatment (Almståhl, Wikström,

\footnotetext{
* Corresponding author at: R. José Bonifácio 1193, 16015-050, Araçatuba, SP, Brazil.

E-mail address: egaettij@foa.unesp.br (E. Gaetti-Jardim).
} 
\& Fagerberg-Mohlin, 2008; Almståh, Wikström, \& Fagerberg-Mohlin, 2015). However, in Brazil, most patients undergoing RT for cancer treatment present low socioeconomic conditions, inadequate oral hygiene standards (Avila-Campos, Ciesielski, \& Sousa, 2011), and dental treatment is not a priority in many radiotherapy centers.

We hypothesized that the occurrence and severity of side effects of radiotherapy could be associated with radiation dose, microbiological aspects, and socioeconomic conditions of the patients. Hence, this study aimed to evaluate RT effects on oral microorganisms and opportunistic pathogens in subgingival and supragingival biofilms from head and neck cancer patients, attended or not by a preventive dental program, correlating the microbiological data with clinical parameters of irradiated patients.

\section{Material and methods}

\subsection{Population and clinical examinations}

This study was approved by the ethics committee of the School of Dentistry of Araçatuba (Process number 01559, UNESP, Araçatuba, SP, Brazil). Patients with head and neck primary malignant neoplasia, with no previous cancer treatment, at least eight natural teeth, not presenting any additional systemic disease and permitting intraoral and extra oral clinical examinations, and who signed the written informed consent were included in this study. All individuals were to start RT at the Regional Unit of Radiotherapy and Megavoltage (São José do Rio Preto, SP, Brazil) or were under clinical follow up at the Oral Oncology Centre (UNESP, Araçatuba, SP, Brazil). Patients with age less than 18 years, receiving bisphosphonates, drugs capable of affecting salivary flow, systemic medication with antimicrobial activity or steroids three months prior to the study, patients presenting history of previous cancer treatment or any pathosis affecting salivary gland function, exhibiting additional systemic diseases or with local factors capable to interfere with periodontal inflammation (excesses in the margins of restorations or crowns) and significant malocclusion were excluded (Almståh et al., 2015; Gaetti-Jardim et al., 2011).

Clinical evaluations involved dental and periodontal assessment with determination of number of teeth, fillings, crowns, bridges, probing depth, gingival recession, bleeding on probing (yes/no), plaque index (presence/absence) along gingival margin, and calculus deposits (Minhas et al., 2016; Gaetti-Jardim et al., 2011). The periodontal attachment loss was calculated by adding the probing depth and recession measurements, determined at six sites per tooth (mesio-buccal, buccal, disto-buccal, mesio-lingual, lingual and disto-lingual). After initial periodontal examinations, bitewing radiographs were taken.

Periodontally healthy subjects were those with absence of gingival inflammation, no bleeding on probing and no radiographic bone loss at all periodontal sites. Patients with gingivitis were those with signs of gingival inflammation involving at least 3 non-contiguous periodontal sites and absence of radiographic bone loss. Patients with chronic periodontitis evidenced attachment loss greater than $5 \mathrm{~mm}$ involving at least 3 non-contiguous periodontal sites and radiographic evidences of bone loss and moderate rate of progression of attachment loss.

The clinical trials were performed before RT, 15-22 days after starting RT, immediately after RT and 6 months after RT (Jham et al., 2007). Patients who eventually gave up treatment, or who could not be contacted, were replaced by other patients as the study progressed. The mean radiation dose received varied from 4320 to 7020 cGy (mean dose 6233 cGy \pm $954.5 \mathrm{cGy}$ ) and the fractioning dose was $180 \mathrm{cGy}$. RT was performed using a linear accelerator and the major salivary glands were lying within the radiation field for all patients (Jham et al., 2007). The most frequent field irradiated was the right and left cervico-facial regions (78.6\%).

Presence and severity of mucositis in patients was determined following the criteria provided by the National Cancer Institute. Summarily: grade 0 is the absence of clinical signs of mucositis; grade I, the mucosa is erythematous and painful; grade II, presence of pseudomembranes less than $1.5 \mathrm{~cm}$ in diameter and the patient feeds orally, consuming solid and semi-solid food; grade III, the presence of pseudomembranes greater than $1.5 \mathrm{~cm}$ in diameter and patient usually feeds liquid diet; and grade IV, evidencing the presence of ulcerations, making oral feeding impossible and requiring parenteral or enteral support.

The occurrence of xerostomia was determined 0by a specific questionnaire (Minhas et al., 2016) and clinical criteria, whereas hyposalivation was evaluated by sialometry (Scarantino et al., 2006). Measurements of salivary flow rates, buffer capacity and saliva $\mathrm{pH}$ were carried out as described by Almståhl et al. (2008). For sialometry of unstimulated whole saliva, all patients were instructed to refrain orofacial movements and to swallow the previous saliva present in their mouths. Then they allowed saliva to accumulate in the floor of mouth for $3 \mathrm{~min}$ and to spit the saliva into a preweighed sterile glass vial. This procedure was repeated more four times to provide data on 15-min collection. The weight of each collection was measured and adjusted to flow in $\mathrm{ml} / \mathrm{min}$ for each patient. Values below $0.1 \mathrm{ml} / \mathrm{min}$ for resting saliva were indicative of hyposalivation.

To determinate the stimulated salivary flow, the stimulated secretion rate was measured using paraffin wax. The first saliva was swallowed and then all saliva secreted during $5 \mathrm{~min}$ was collected. This saliva was used to measure the $\mathrm{pH}$ and buffer capacity, using a pH/ion meter (Tecnopon mPA210, Piracicaba, SP, Brazil). Buffer capacity of saliva was determined according to Ericsson's method (1959) (Ericsson, 1959).

Initially, a total of twenty-eight patients (20 males and 8 females) out of 183 patients, aged 18-68 years (mean age: 56.2 years), were included in this study. These patients harbored at least eight teeth (mean: $12.8 \pm 4.5$ ). Two subjects were periodontally healthy, ten had chronic periodontitis and sixteen presented gingivitis. Regarding the diagnosis of malignancy, twenty-six patients presented squamous cell carcinoma, one patient harbored acinar cell carcinoma and one presented basal cell carcinoma. Most patients were illiterate or had elementary formal education (85.7\%), and were tobacco (92.9\%) and/or alcohol (71.4\%) consumers. The head and neck cancer treatment consisted of RT alone (42.9\%) or combined with chemotherapy $(10.7 \%)$, surgical resection (35.7\%), or surgery and chemotherapy $(10.7 \%)$. Patients were instructed to seek dental care before starting RT, but only $32.1 \%$ received dental treatment (dental extractions, periodontal treatment, and restorative procedures).

Each patient undergoing RT was matched according to age, sex, alcohol and tobacco consumption, periodontal and oral hygiene conditions with a control group with normal salivary flow rates and no history of head and neck cancer or cancer treatment. The control group patients were volunteers ( 18 males and 10 females) from the general public seen at the School of Dentistry of Araçatuba (UNESP, Araçatuba, SP, Brazil) aged 18-65 years (mean age: 52.4 years), harboring $14.2 \pm 5.3$ natural teeth; three were periodontally healthy; nine had periodontitis and sixteen presented gingivitis. All control group subjects presented an unstimulated salivary flow rate $>0.1 \mathrm{ml} / \mathrm{min}$ and a stimulated salivary flow rate $>1.0 \mathrm{ml} / \mathrm{min}$ and these values were considered normal (Almståhl et al., 2008).

After clinical examinations, patients received oral care instructions by dentists. In order to prevent radiation caries, patients were instructed to follow a protocol consisting of twice-daily use of $1100 \mathrm{ppm}$ sodium fluoride toothpaste (Colgate-Palmolive Co., Sao Paulo, SP, Brazil) and daily aqueous fluoride $(0.05 \% \mathrm{NaF})$. All irradiated patients who developed oral candidiasis were instructed to rinse oral mucosa with $5 \mathrm{ml}$ of an oral suspension containing 100,000 U/ml aqueous nystatin three times a day (Almståhl et al., 2008) (Micostatin ${ }^{\circledR}$, Bristol Myers Squibb, Sao Paulo, SP, Brazil).

\subsection{Clinical samples}

Subgingival and supragingival biofilm samples were collected before clinical examinations. Subgingival samples of the three most 
diseased periodontal sites of each patient were collected using sterilized paper points, introduced into the apical region of the periodontal pockets or gingival crevice and allowed to remain for $60 \mathrm{~s}$. The paper points were then pooled and transferred to VGMA III transport medium. Collection of the subgingival biofilm was carried out after removal of the supragingival biofilm with periodontal curettes and samples were also transferred to VMGA III.

\subsection{Microbial isolation and identification}

Clinical specimens transported in VMGA III medium were submitted to serial 10-fold dilutions in VMGA I and plated onto fastidious anaerobe agar (FAA) enriched with $5 \mathrm{mg} / \mathrm{ml}$ hemin, $1 \mathrm{mg} / \mathrm{ml}$ menadione, $0.5 \%$ yeast extract, $10 \mathrm{mg} / \mathrm{ml} \mathrm{N}$-acetyl-muramic acid and $5 \%$ sheep blood; Mitis Salivarius agar (Difco, Detroit, MI, USA); Mitis-SalivariusBacitracin agar, CFAT agar; TSBV agar, and incubated in anaerobiosis $\left(90 \% \mathrm{~N}_{2}+10 \% \mathrm{CO}_{2}\right)$ at $37^{\circ} \mathrm{C}$, for $10,2,2,3$, and 3 days, for obligate anaerobes, oral streptococci, mutans streptococci, actinomycetes, and Aggregatibacter actinomycetemcomitans.

These samples were also inoculated onto tryptic soy agar supplemented with 5\% sheep blood and $0.5 \%$ yeast extract; Mannit Salt agar (Eiken, Tokyo, Japan); Sabouraud dextrose agar with $100 \mu \mathrm{g} / \mathrm{ml}$ chloramphenicol, and incubated at $37^{\circ} \mathrm{C}$ for $48-72 \mathrm{~h}$, for aerobic and facultative anaerobic microorganisms. Additionally, $0.1 \mathrm{ml}$ of clinical specimens was transferred to peptone water and ethyl violet azide broth (EVA broth), incubated at room temperature for $72 \mathrm{~h}$. Aliquots from tubes with microbial growth were inoculated onto Eosin-Methylene Blue agar, SS agar, Brilliant Green agar and incubated at $37^{\circ} \mathrm{C}$, for $48 \mathrm{~h}$ (Monti, Ciesielski, Gaetti-Jardim, Okamoto, \& Schweitzer, 2012).

Bacterial identification was carried out by Gram staining, colony morphology, and by commercial kits as previously described (GaettiJardim et al., 2011; Gaetti-Jardim et al., 2012). The yeast identification was performed by colonial morphology and pigmentation on CHROM agar (MastDiagnostica, Paris, France), carbon and nitrogen assimilation tests, fermentation of carbohydrates, germ tube formation (at $37^{\circ} \mathrm{C}$ and at $39^{\circ} \mathrm{C}$ ), and growth at $37^{\circ} \mathrm{C}$ and $42{ }^{\circ} \mathrm{C}$ (Freitas et al., 2013). Isolates of genera Actinomyces, Porphyromonas and Prevotella were also specified through DNA amplification by polymerase chain reaction (PCR) using specific primer pairs (Freitas et al., 2013).

Concerning microbiological data, only results of microorganisms isolated from at least $10 \%$ irradiated patients and at least 3 of the 4 sample collections were presented.

\section{Statistical analysis}

All statistical analyses were performed using the Statistica Software 7.0, and MS Excell software, which was employed in the descriptive statistics (means, standard deviations, frequencies, and percentages), presented through of graphics and tables. Differences between variables with three or more categories were submitted to Chi-square test. Interrelationships between clinical and microbiological parameters were assessed by Fisher and Mann-Whitney's exact test; changes in the distribution of different microorganisms were evaluated by analysis of variance with repeated measures for categorical data, ANOVArm (Brunner \& Langer, 2000). Changes in microbial populations in the biofilms were evaluated by Kruskal-Wallis analysis of variance (ANOVA). Differences of $p<0.05$ were considered statistically significant.

\section{Results}

The occurrence of mucositis, dermatitis, xerostomia, dysgeusia, dysphagia and, to a lesser extent, candidiasis was common in irradiated patients, even 6 months after completion of RT. Immediately after completion of RT, $57.1 \%$ presented grade III or IV mucositis. Severe mucositis was not reported 6 months after RT, but 53.6\% patients still presented moderate or mild disease (grade I or II). The severity of mucositis was associated with xerostomia $(p=0.016)$, poor oral hygiene $(p<0.001)$, oral candidiasis $(p=0.02)$, and absence of preventive oral care $(p=0.002)$. Gender, formal education, amount of alcohol or tobacco consumption, dental caries, and periodontal condition did not interfere with severity of mucositis.

Candidiasis, dermatitis, dysphagia, dysgeusia, mucositis and xerostomia were not detected before RT. Immediately after completion of RT, these conditions were detected in $42.9 \%, 82.1 \%, 32.1 \%, 75 \%$, $85.7 \%$, and $89.3 \%$ of patients, respectively. Six months after completion of RT, candidiasis (14.3\%), dermatitis (21.4\%), dysphagia (17.9\%), dysgeusia (35.7\%), and xerostomia (53.6\%) were still observed. It was noted that RT was also associated with a drop in salivary $\mathrm{pH}$ $(p=0.023)$ and reduction of buffer capacity of saliva $(p=0.019)$, as presented in Table 1.

The oral hygiene conditions were generally poor before RT, similar to control group patients (Table 1 ), but evidenced severe deterioration during and after RT $(p=0.042)$. Before RT, only $10.7 \%$ had good hygiene (absence of visible biofilm), while $39.3 \%$ presented moderate

Table 1

Clinical periodontal conditions, salivary flow, buffer capacity of patients submitted to radiotherapy for head and neck cancer treatment (mean values and standard deviation).

\begin{tabular}{|c|c|c|c|c|c|c|c|c|}
\hline \multirow[t]{2}{*}{ Parameters } & \multicolumn{2}{|l|}{ Stage $1^{\mathrm{a}}$} & \multicolumn{2}{|l|}{ Stage $2^{\mathrm{b}}$} & \multicolumn{2}{|l|}{ Stage $3^{\mathrm{c}}$} & \multicolumn{2}{|l|}{ Stage $4^{\mathrm{d}}$} \\
\hline & $\begin{array}{l}\text { Irradiated } \\
\text { Patients }\end{array}$ & Control Group & $\begin{array}{l}\text { Irradiated } \\
\text { Patients }\end{array}$ & Control Group & $\begin{array}{l}\text { Irradiated } \\
\text { Patients }\end{array}$ & Control Group & $\begin{array}{l}\text { Irradiated } \\
\text { Patients }\end{array}$ & Control Group \\
\hline $\begin{array}{l}\text { Surfaces with plaque along } \\
\text { gingival margin (\%) }\end{array}$ & $52.5 \pm 10.2$ & $49.2 \pm 18.2$ & $64.5 \pm 16.2^{*}$ & $45.2 \pm 19.3$ & $60.1 \pm 13.2^{*}$ & $44.2 \pm 16.5$ & $61.5 \pm 18.5^{*}$ & $47.4 \pm 16.3$ \\
\hline $\begin{array}{l}\text { Surfaces with bleeding on } \\
\text { probing }(\%)\end{array}$ & $33.7 \pm 20.2$ & $31.5 \pm 16.3$ & $44.2 \pm 14.2^{k}$ & $29.8 \pm 18.1$ & $42.1 \pm 16.4$ & $30.3 \pm 15.7$ & $45.3 \pm 17.2^{*}$ & $31.4 \pm 18.4$ \\
\hline Probing depth (mm) & $3.3 \pm 2.6$ & $3.5 \pm 1.8$ & $3.5 \pm 2.2$ & $3.4 \pm 1.7$ & $3.6 \pm 2.5$ & $3.7 \pm 1.7$ & $3.5 \pm 2.7$ & $3.4 \pm 2.2$ \\
\hline $\begin{array}{l}\text { Unstimulated secretion rate } \\
\qquad(\mathrm{ml} / \mathrm{min})\end{array}$ & $0.6 \pm 0.2$ & $0.5 \pm 0.2$ & $0.07 \pm 0.03^{*}$ & $0.7 \pm 0.2$ & $0.02 \pm 0.01^{*}$ & $0.6 \pm 0.3$ & $0.04 \pm 0.03^{*}$ & $0.6 \pm 0.4$ \\
\hline $\begin{array}{l}\text { Stimulated secretion rate }(\mathrm{ml} / \\
\min )\end{array}$ & $2.4 \pm 1.3$ & $2.5 \pm 1.2$ & $0.45 \pm 0.41^{k}$ & $2.7 \pm 1.4$ & $0.3 \pm 0.28^{*}$ & $2.2 \pm 1.7$ & $0.5 \pm 0.39^{*}$ & $2.3 \pm 1.7$ \\
\hline $\mathrm{pH}$ & $7.1 \pm 0.6$ & $7.3 \pm 0.7$ & $6.2 \pm 0.9^{*}$ & $7.0 \pm 0.5$ & $6.0 \pm 0.6^{*}$ & $6.9 \pm 0.9$ & $6.1 \pm 0.7^{*}$ & $7.2 \pm 0.8$ \\
\hline Buffer capacity & $5.5 \pm 0.8$ & $5.9 \pm 0.7$ & $4.1 \pm 0.7^{*}$ & $5.7 \pm 0.9$ & $3.9 \pm 0.6^{*}$ & $5.6 \pm 0.7$ & $3.7 \pm 0.8^{*}$ & $5.5 \pm 0.5$ \\
\hline
\end{tabular}

* Significant difference, $\mathrm{p}<0.05$.

a Stage 1: before RT.

b Stage 2, 15-22 days after beginning of RT.

c Stage 3, immediately after RT.

${ }^{\mathrm{d}}$ Stage 4, 6 months after completion of RT. 
hygiene (less than $50 \%$ of teeth surfaces covered with microbial biofilm), and $50 \%$ poor hygiene conditions (extensive calculus deposits on teeth, food residues, and more than $50 \%$ of teeth surfaces covered with microbial biofilm). After completion of RT, good, moderate and poor hygiene conditions were seen in $3.6 \%, 21.4 \%$ and $75 \%$ of patients, respectively. After the 6 months of follow-up period, good, moderate and poor hygiene was detected in $3.6 \%, 32.1 \%$ and $64.3 \%$ of irradiated subjects, respectively.

Initial periodontal parameters (bleeding on probing and probing depth) of irradiated patients were similar to those reported by control group patients and RT did not significantly change the periodontal status of patients $(p=0.179)$.

In the control group, there were no quantitative or qualitative significant changes in the microbiota in the analyzed period and the microbiological data from oncologic patients in the period preceding RT were similar to those observed for the control group.

The occurrence of different microbial species and genera in the supragingival biofilm of irradiated patients throughout the study are shown in Fig. 1. The occurrence of $A$. odontolyticus ( $p=0.045), A$. viscosus $(p=0.025), C$. albicans $(p=0.027)$, non-albicans Candida $(p=0.032), C$. ochracea $(p=0.028)$, E. corrodens $(p=0.042), E$. faecalis $(p=0.033), P$. micra $(p=0.031), S$. intermedius $(p=0.037), S$. mitior $(p=0.04)$ and $S$. sobrinus $(p=0.028)$ increased during RT, whereas the prevalence of. A. naeslundii $(p=0.03), P$. aeruginosa $(p=0.043), P$. nigrescens $(p=0.027)$, and family Enterobacteriaceae $(p<0.001)$ rose particularly after RT. The occurrence of $C$. albicans and non-albicans Candida in the oral biofilm suffered significant changes along RT, particularly in the supragingival biofilm (before RT: C. albicans, 25\%; C. glabrata, 14.3\%, C. tropicalis, 3.6\%; C. parapsilosis, 3.6\%; 6 months after completion of RT: C. albicans, 82.1\%; C. glabrata, 21.4\%; C. tropicalis, 10.7\%; C. dubliniensis, 10.7\%; C. kefyr, 3.6\%, C. parapsilosis, $3.6 \%$ and C. krusei, 3.6\%).

Concerning the quantitative aspects of the supragingival biofilm (Table 2), microbial populations remained stable $(p=0.178)$ during

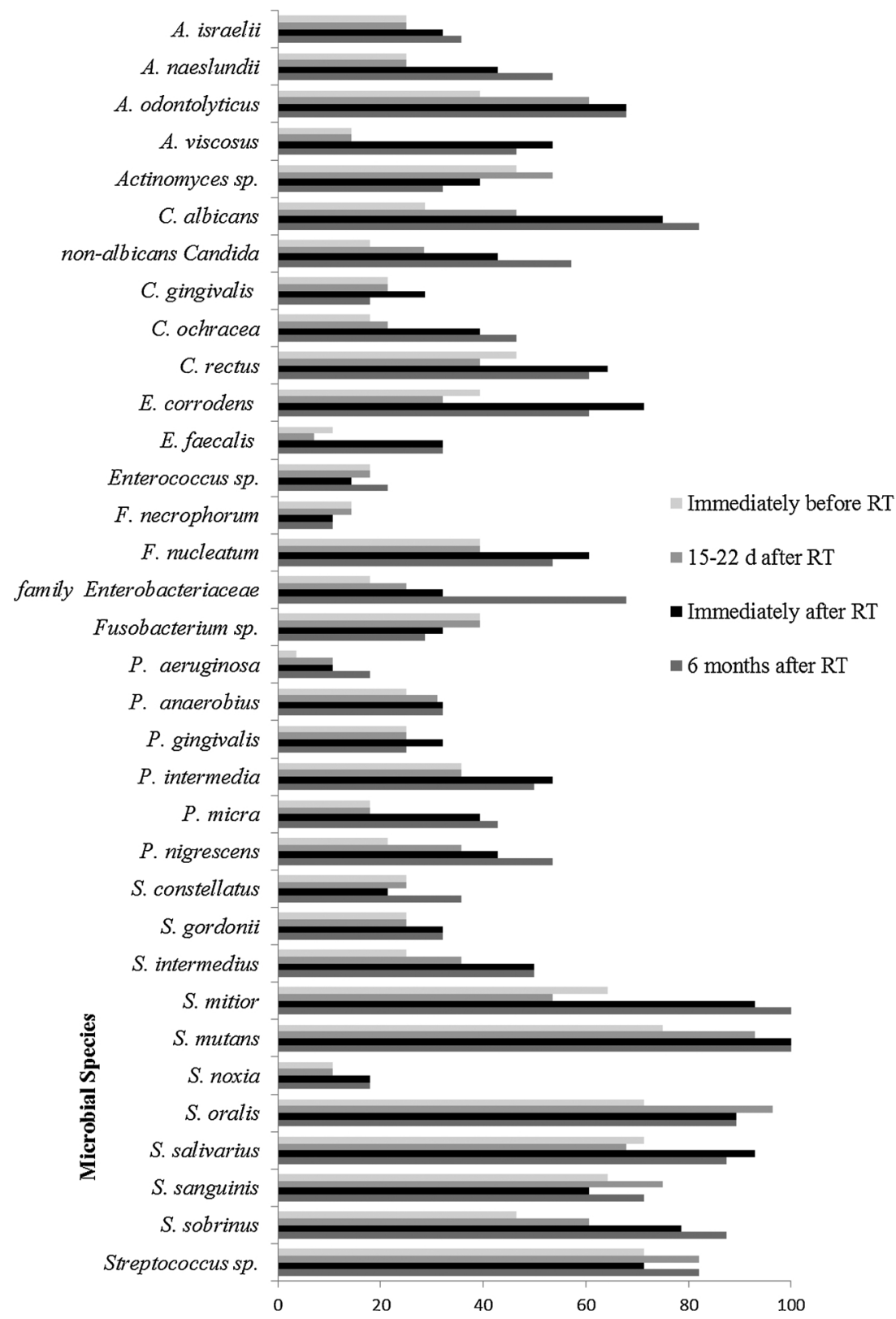

Fig. 1. Occurrence of different species and bacterial groups in supragingival biofilm of irradiated patients. 
Table 2

Populations of different microorganisms in supragingival biofilm of irradiated patients along time.

\begin{tabular}{|c|c|c|c|c|}
\hline Microbial counts $(\log 10)$ & Before RT & $15-22 \mathrm{~d}$ from beginning of RT & After RT & 6 months after RT \\
\hline Actinomycetes & $5.33 \pm 0.28$ & $5.9 \pm 0.33^{*}$ & $5.7 \pm 0.51^{*}$ & $6.01 \pm 0.28^{*}$ \\
\hline Candida spp. & $0.61 \pm 0.52$ & $1.38 \pm 0.39^{*}$ & $2.2 \pm 0.8^{*}$ & $3.1 \pm 0.46^{*}$ \\
\hline Enterococcus spp. & $1.7 \pm 0.43$ & $1.62 \pm 0.41$ & $2.02 \pm 0.61^{*}$ & $2.6 \pm 0.39^{*}$ \\
\hline family Enterobacteriaceae & $2.72 \pm 0.31$ & $2.58 \pm 0.42$ & $3.88 \pm 0.45^{*}$ & $3.47 \pm 0.39^{\prime \prime}$ \\
\hline F. nucleatum & $5.06 \pm 0.34$ & $5.11 \pm 0.54$ & $5.24 \pm 0.61$ & $5.1 \pm 0.38$ \\
\hline mutans streptococci & $5.1 \pm 0.41$ & $6.32 \pm 0.49^{*}$ & $6.41 \pm 0.57$ & $6.1 \pm 0.43^{*}$ \\
\hline Total streptococci & $6.01 \pm 0.33$ & $6.2 \pm 0.44$ & $5.7 \pm 0.49$ & $5.9 \pm 0.37$ \\
\hline Parvimonas micra & $3.89 \pm 0.57$ & $3.22 \pm 0.61$ & $3.04 \pm 0.56$ & $3.57 \pm 0.39$ \\
\hline Porphyromonas gingivalis & $3.12 \pm 0.35$ & $2.32 \pm 0.41^{* * *}$ & $2.02 \pm 0.53^{k * k}$ & $2.19 \pm 0.58^{* k}$ \\
\hline Prevotella intermedia/nigrescens & $4.48 \pm 0.48$ & $3.57 \pm 0.65^{* *}$ & $2.97 \pm 0.34^{* *}$ & $2.7 \pm 0.37^{* *}$ \\
\hline Total microbial counts & $7.5 \pm 0.46$ & $7.18 \pm 0.62$ & $7.62 \pm 0.41$ & $7.02 \pm 0.61$ \\
\hline
\end{tabular}

* Significantly higher values than those observed in control group and in initial values, before RT.

** Significantly lower values than those observed in control group and in initial values, before RT.

and after RT. There was an increase in populations of actinomycetes during the initial period of RT ( $p=0.043$ ), which remained stable until the end of the follow-up; enterococci $(p=0.019)$, after RT; family Enterobacteriaceae ( $p=0.026)$, at completion of RT; and mutans streptococci $(p=0.033)$, throughout the experiment. Of the tested microorganisms, the effects of RT were more intense on populations of genus Candida $(p=0.01)$. Supragingival populations of mutans streptococci $(p=0.028)$, yeasts $(p=0.021)$ and family Enterobacteriaceae $(\mathrm{p}=0.0013)$ were also associated with xerostomia. After completion of RT, incidence and populations of such microorganisms remained higher than before treatment. RT was also associated with reduction of populations of anaerobic Gram-negative rods, particularly $P$. gingivalis $(p=0.043)$ and Prevotella intermedia/P. nigrescens $(p=0.037)$ in the supragingival biofilm.

In the subgingival biofilm (Fig. 2), the occurrence of $A$. odontolyticus ( $p=0.037)$, Candida spp. $(p=0.029), P$. endodontalis $(p=0.028), P$. intermedia $(p=0.03), P$. nigrescens $(p=0.03), S$. mutans $(p=0.042)$, S. sobrinus $(p=0.019), \quad S$. gordonii $(p=0.03)$, and family $E n-$ terobacteriaceae $(p<0.001)$ increased significantly during and after RT. Changes in the subgingival biofilm proved to be slower than those observed in the supragingival biofilm.

Family Enterobacteriaceae evidenced the most significant increase, throughout the experiment, also associated with xerostomia $(p=0.04)$. Quantitative changes in the subgingival biofilm (Table 3 ) were similar to those reported for the supragingival biofilm. Populations of family Enterobacteriaceae $(p=0.023), P$. gingivalis $(p=0.034)$ and $P$. intermedia, $P$. nigrescens $(p=0.04)$ increased after completion of RT, and populations of Candida species increased gradually over RT and remained higher until the end of the follow-up period $(p=0.026)$, and were also correlated with poor hygiene standards $(p=0.036)$.

The occurrence of family Enterobacteriaceae in the supra $(\mathrm{p}=0.021)$ and subgingival biofilms $(\mathrm{p}=0.04)$ was also associated with tobacco use. Family Enterobacteriaceae and Candida spp. were associated with mucositis grade III or IV $(p=0.035)$. For family Enterobacteriaceae no species was individually significant, but the changes in their distribution and populations were collectively significant. In the supragingival biofilm before RT, the most frequently isolated members were Enterobacter cloacae (7.1\%), Escherichia coli (7.1\%), Klebsiella pneumoniae (7.1\%), and Proteus sp. (7.1\%), which were recovered from $17.9 \%$ patients. In the subgingival biofilm before RT, E. cloacae $(10.7 \%), K$. oxytoca (3.6\%), and P. mirabilis (3.6\%) were also isolated from $17.9 \%$ patients.

Six months after completion of RT, 21 species of this family were isolated, particularly from the supragingival biofilm. In these samples, C. freundii (14.3\%), E. cloacae (10.7\%), P. mirabilis (10.7\%), P. rettgeri $(10.7 \%)$, and $S$. liquefaciens $(7.1 \%)$ were recovered from $67.9 \%$ patients, whereas in the subgingival biofilm, C. freundii (10.7\%), E. cloacae (14.3\%), E. sakazakii (10.7\%), M. morganii (10.7\%), P. agglomerans
(7.1\%), P. mirabilis $(10.7 \%)$, P. vulgaris $(7.1 \%)$, P. rettgeri $(7.1 \%)$ and Serratia sp. (7.1\%) were isolated from $60.7 \%$ patients.

\section{Discussion}

RT side effects significantly impacted the quality of life of irradiated patients, in such a way that preventive dental care must be introduced early and maintained during and after RT (Almståh et al., 2015; Leung, Jin, Yam, \& Samaranayake, 2001; Schuurhuis et al., 2016; Sun, Gao, Deng, Li, \& Lu, 2010). In the present study, the major patient complaint was the incidence of mucositis, which developed soon after the first two weeks of RT, frequently associated with xerostomia and candidiasis (Freitas et al., 2013). Mucositis severity was correlated with presence of xerostomia, absence of prior dental treatment, oral candidiasis, poor hygiene and colonization of supra and subgingival biofilms by members of family Enterobacteriaceae and genus Candida.

Radiotherapy-induced mucositis is dosage-dependent, but inflammation regresses after conclusion of RT more promptly than the severity of xerostomia (Sun et al., 2010). Although the present study showed a correlation between Gram-negative enteric rods and mucositis grade III and IV, the role that the microbiota plays in the development and aggravation of mucositis needs further evaluation. It is suggested that this oral condition can be exacerbated by the presence of other local infectious and/or systemic processes (Vanhoecke, De Ryck, Stringer, Van de Wiele, \& Keefe, 2015).

Data from the present study showed that the prevalence and populations of these yeasts did not differ between RT and control group at the beginning of the experiment. RT showed a direct correlation with the increase of prevalence and populations of Candida genus along the entire study period, in agreement with the literature (Sonalika et al., 2012; Vanhoecke et al., 2015).

Patients who initially presented the highest populations of these yeasts were also the first ones to develop clinically detectable infection, as observed by Jham et al. (2007) and Gaetti-Jardim et al. (2011). The initial colonization by these yeasts may have a predictive value on incidence and severity of fungal infection (Jham et al., 2007). The prevalence of these yeasts may also reflect the precarious oral hygiene condition of most patients and its deterioration along RT, as suggested by Yogitha, Lakshmi, Lakshmi, Krishna, and Cheemala (2015). These authors also observed a correlation between the presence of these yeasts and nutritional deficits/weight loss in irradiated patients, a frequent phenomenon in the studied patients.

The initial detection frequencies of $C$. albicans and non-albicans Candida were similar to the data of Jham et al. (2007), but higher than those obtained with irradiated patients submitted to more comprehensive preventive programs (Schuurhuis et al., 2016; Yogitha et al., 2015). Despite evidences that suggest a progressive and more significant increase in the distribution of non-albicans Candida (Bakki 


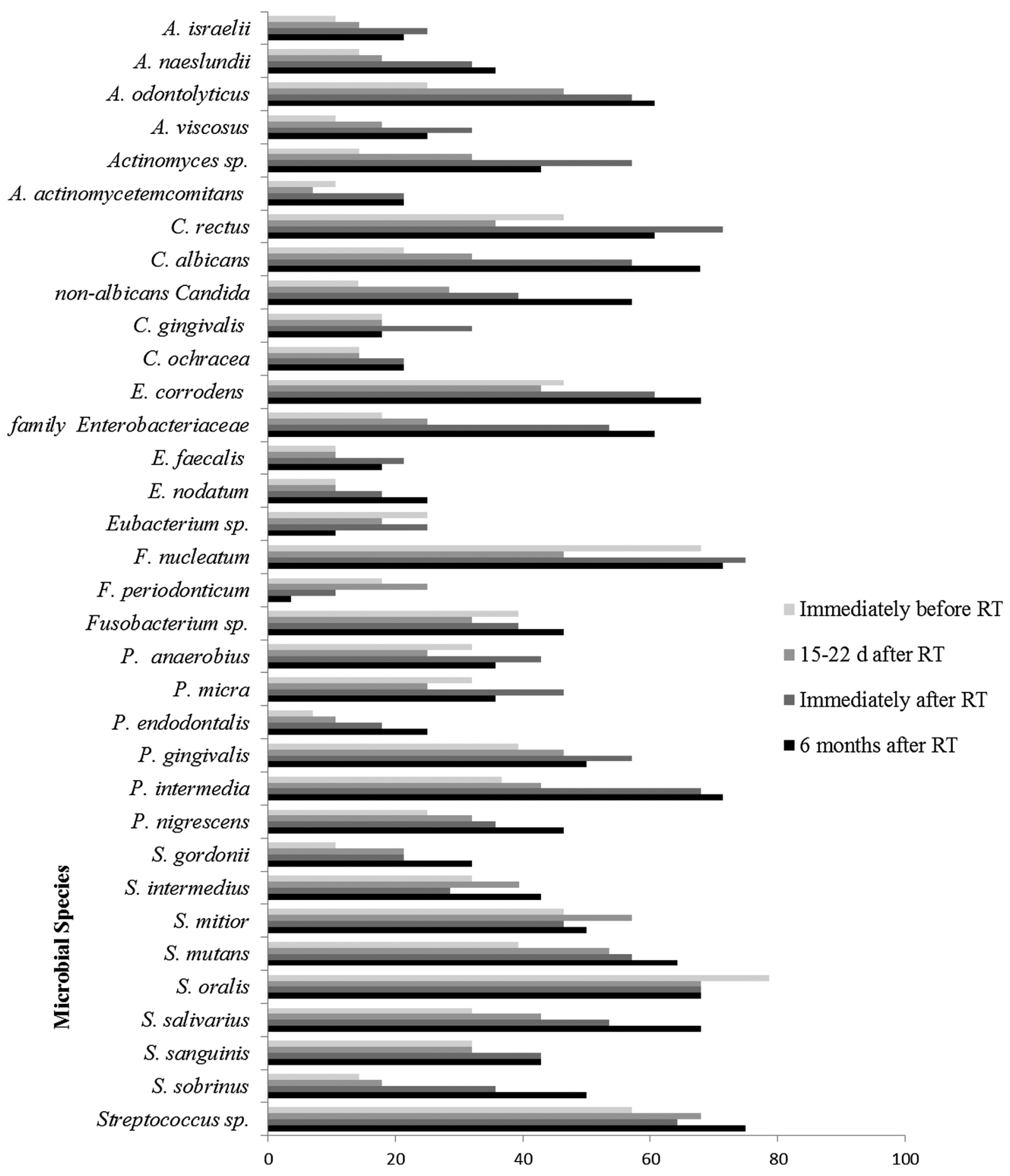

Fig. 2. Occurrence of different species and bacterial groups in subgingival biofilm of irradiated patients.

et al., 2014; Jham et al., 2007), the results of Tables 1 and 2 show that RT favored most Candida genera, indistinctively, as previously observed (Sonalika et al., 2012; Yogitha et al., 2015), even after completion of RT treatment (Yogitha et al., 2015).

The aggravation of virulence of these yeasts, as well as reduction of salivation are definitely involved in the development of oral candidiasis and, possibly, aggravation of mucositis (Bakki et al., 2014). Therefore, prevention/control of candidiasis, through improvement of oral hygiene conditions, use of antifungal solutions and nutritional support might constitute an adequate measure to control the severity of mucositis in irradiated patients (Jham et al., 2007; Yogitha et al., 2015).

Salivary flow reduction, buffer capacity, as well as acidification of the oral environment seem to be associated not only with the significant increase in occurrence and populations of yeasts, but also of cariogenic cocci (Meng et al., 2005). Nevertheless, additional caries lesions were not reported, which might be associated with the effectiveness of the sodium fluoride solution used in the preventive protocol. On the other hand, the relatively short patient follow-up period would not allow a more thorough evaluation, as previously reported (Sun et al., 2010).

The data concerning RT effects on occurrence and populations of cariogenic streptococci are inconsistent. Some authors showed transitory increase of these microbial populations or no significant effects (Eliasson, Carlén, Almståhl, Wikström, \& Lingström, 2006), whereas patients with poor hygiene conditions supported a significant increase 
Table 3

Populations of different microorganisms in subgingival biofilm of irradiated patients along time.

\begin{tabular}{|c|c|c|c|c|}
\hline Microbial counts $(\log 10)$ & Before RT & 15-22d from beginning of RT & After RT & 6 months after RT \\
\hline Actinomycetes & $2.89 \pm 1.31$ & $3.01 \pm 0.43$ & $3.2 \pm 0.28$ & $2.9 \pm 1.06$ \\
\hline Candida spp. & $0.27 \pm 0.33$ & $1.18 \pm 0.46^{*}$ & $2.6 \pm 0.54^{*}$ & $2.7 \pm 0.49^{*}$ \\
\hline Enterococcus spp. & $0.83 \pm 0.55$ & $0.72 \pm 0.39$ & $0.9 \pm 0.42$ & $0.78 \pm 0.57$ \\
\hline family Enterobacteriaceae & $2.1 \pm 0.37$ & $2.09 \pm 0.48$ & $2.91 \pm 0.72$ & $3.25 \pm 0.56^{\prime \prime}$ \\
\hline F. nucleatum & $5.2 \pm 0.39$ & $4.89 \pm 0.42$ & $5.24 \pm 0.36$ & $5.1 \pm 0.32$ \\
\hline mutans streptococci & $2.4 \pm 0.81$ & $3.1 \pm 0.55^{*}$ & $3.9 \pm 1.14$ & $4.0 \pm 0.63$ \\
\hline Total streptococci & $4.1 \pm 0.56$ & $4.4 \pm 0.71$ & $4.51 \pm 0.63$ & $4.61 \pm 0.57$ \\
\hline Parvimonas micra & $3.27 \pm 0.46$ & $3.54 \pm 0.41$ & $3.31 \pm 0.71$ & $3.43 \pm 0.51$ \\
\hline Porphyromonas gingivalis & $3.41 \pm 0.52$ & $3.67 \pm 0.39$ & $4.72 \pm 0.43^{*}$ & $4.87 \pm 0.37$ \\
\hline Prevotella intermedia/nigrescens & $3.48 \pm 0.55$ & $3.61 \pm 0.62$ & $4.49 \pm 0.44^{*}$ & $4.9 \pm 1.07^{*}$ \\
\hline Total microbial counts & $6.2 \pm 0.53$ & $6.43 \pm 0.81$ & $5.91 \pm 0.65$ & $6.41 \pm 0.57$ \\
\hline
\end{tabular}

* Significantly higher values than those observed in control group and in initial values, before RT.

of populations of these acidogenic cocci (Meng et al., 2005), as reported in the present study. RT effects on these streptococci seem to be cumulative, accompanying the severity of xerostomia, which normally sets after 2000 cGy of radiation (Sun et al., 2010). However, the use of sodium fluoride solution may have minimized the increase of these microorganisms, contributing to reduce the occurrence of caries associated with xerostomia (Almståhl et al., 2008). Although an association between the increase in oral streptococci populations and mucositis severity has not been observed, $S$. anginosus, $S$. mitis, $S$. mutans, $S$. oralis, $S$. sanguinis, and $S$. sobrinus present mucolytic capacity for the production of glycosidases and may aggravate mucositis, leading to changes in composition and thickness of the oral mucus (Vanhoecke et al., 2015).

The present study showed that RT caused less significant interferences in the distribution of microaerophiles and obligate anaerobes of supra and subgingival biofilms, when compared with radiation effects on yeasts, cariogenic cocci and coliforms, as also previously reported (Sonalika et al., 2012). Although the prevalence and populations of most obligate anaerobe and microaerophilic microbial groups were constant along the study, populations of Gram-negative anaerobes of Porphyromonas and Prevotella genera decreased in the supragingival biofilm but increased in the gingival sulcus of patients who presented gingivitis or chronic periodontitis and significant oral hygiene deterioration, even between those who received oral treatment prior to RT.

Severity and duration of xerostomia seem to act as determining factors of biofilm composition changes, mainly in the supragingival environment. Schuurhuis et al. (Schuurhuis et al., 2016) analyzed patients with less severe xerostomia due to the type of RT adopted, associated with oral preventive procedures to control infection foci, and revealed consistent reduction of the populations of the main periodontopathogens along RT. Probably, the periodontal conditions of those patients and the received preventive care were more favorable than in the present study.

Poor oral hygiene conditions and presence of gingivitis or periodontitis in almost all patients submitted to RT may have corroborated for an elevated occurrence of Actinomyces, Capnocytophaga, Eikenella, Fusobacterium, Prevotella and Porphyromonas genera, reinforcing the need for improvement of preventive measures. Besides this aspect, mucositis ulcerated lesions significantly impaired oral hygiene in most patients, even in those who received prior dental care, since the treatment was limited to exodontias and dental restorations, interfering little with the biofilm and hygiene conditions.

The presence of intense gingival bleeding is frequently associated with xerostomia (Gonçalves et al., 2007), as also noticed in the present study. Nevertheless, based on evidences suggesting that periodontitis occurrence is higher between irradiated and mucositis patients (Mizutani et al., 2015), further longitudinal and extensive studies are necessary with patients without oral preventive care. It is important to verify this possible relation, but also to evaluate the impact of preventive care on the periodontal health of irradiated patients. It is possible to suggest that the appearance of ulceration and lesions near the gingival sulcus creates a more irregular surface, potentiating microbial adhesion and increasing the presence of the same pathogens present in the biofilm of oropharyngeal carcinoma patients.

The increase of the populations of the microorganisms associated with periodontitis, such as Gram-negative anaerobes in subgingival biofilm could be due to vascular changes induced by RT, with blood supply reduction and, possibly, decrease of the redox potential of the periodontal tissues. However, the populations of these anaerobes were decreased in the supragingival biofilm, which suffers the effects of xerostomia and oral condition changes of patients more intensely can be associated to the acidification of the environment due to proliferation of acidogenic cocci and by reduction of the saliva buffer capacity in patients with xerostomia.

Some of these anaerobes and family Enterobacteriaceae up-regulate cytokines and inflammatory chemokines release (Vanhoecke et al., 2015), aggravating the initial acute effects of mucositis. Accordingly, the nearly universal presence of these microorganisms in the subgingival and supragingival biofilms of irradiated patients impairs associations with specific oral conditions and may reflect the deterioration of hygiene conditions along RT treatment, although secondary contamination of lesions may worsen symptoms.

The most significant changes in prevalence and microbial populations were observed in members of the family Enterobacteriaceae, buccal yeasts (Almståhl et al., 2008; Gaetti-Jardim et al., 2011; Leung et al., 2001; Schuurhuis et al., 2016), and for P. aeruginosa (Almståhl et al., 2008; Gaetti-Jardim et al., 2011; Schuurhuis et al., 2016), involved with nosocomial multi-resistant infections and frequently present in the subgingival biofilm of patients with periodontitis in Brazil (Gonçalves et al., 2007). The occurrence of these microorganisms in the microbial biofilm is associated with poor hygiene and oral conditions (Schuurhuis et al., 2016), as well as alcohol and tobacco consumption, which facilitate adhesion and oral colonization by enteric microorganisms in irradiated patients (Gaetti-Jardim et al., 2011; Sonalika et al., 2012). Among the evaluated patients, only $7.1 \%$ did not declare themselves as alcohol and/or tobacco consumers.

According to Sonalika et al. (Sonalika et al., 2012), the fimbriae of these microorganisms may facilitate microbial adhesion to tumoral cells, increasing the colonization of head and neck malignant neoplastic lesions, mainly in irradiated patients with xerostomia, and the presence of these microorganisms in the surface of oral soft tissues might aggravate microbial proteolytic activity on fibronectin, exposing adhesion receptors to members of the family Enterobacteriaceae. This process can intensify radiotherapy-induced mucositis by inducing inflammation through direct LPS action and interleukins Il-1 and Il-6 release.

This oral colonization by pseudomonads and family Enterobacteriaceae may be transitory (Leung et al., 2001) or stable (Gaetti-Jardim et al., 2011), possibly by maintaining the factors that facilitate initial colonization, aggravated by malnutrition and weight loss which follow dysphagia, a frequent characteristic in irradiated patients. 
Patients analyzed in the present study exhibited poor hygiene and more severe periodontal impairment when compared with other irradiated patients (Almståh et al., 2015; Leung et al., 2001; Schuurhuis et al., 2016). In addition, low compliance to diet and hygiene instructions was observed, as well as modest adhesion to preventive dental programs before RT. Thus, although Schuurhuis et al. (2016) showed a reduction in the diversity of the oral microbiota with RT, this phenomenon seems to be limited to patients who received extensive dental care and presented satisfactory general hygiene prior to RT, which do not correspond to the reality of most patients attended in regions of the interior of Brazil, where dental care is not always considered a priority by the patient and health team.

\section{Conclusions}

The results confirm that RT can induce significant changes in the distribution and populations of some microorganisms, mainly in the supragingival biofilm. The occurrence and populations of yeasts of genus Candida and species of family Enterobacteriaceae increased most significantly and were related to oral hygiene deterioration, tobacco consumption and mucositis severity. These microorganisms were also more common among patients who did not receive oral treatment prior to RT.

\section{Conflict of interests}

There is not conflict of interests.

\section{Ethical approval}

This study was approved by the ethics committee of the School of Dentistry of Araçatuba (Process number 01559, UNESP, Araçatuba, SP, Brazil).

\section{Acknowledgement}

This study was partially supported by grants from the Sao Paulo Research Foundation (FAPESP, Proc. 07/54851-0 and 13/056650-3).

\section{References}

Almståh, A., Wikström, M., \& Fagerberg-Mohlin, B. (2015). Microflora in oral ecosystems and salivary secretion rates: A 3-year follow-up after radiation therapy to the head and neck region. Archives of Oral Biology, 60, 1187-1195.

Almståhl, A., Wikström, M., \& Fagerberg-Mohlin, B. (2008). Microflora in oral ecosystems in subjects with radiationinduced hyposalivation. Oral Diseases, 14, 541-549.

Bakki, S. R., Kantheti, L. P., Kuruba, K. K., Poosarla, C., Baddam, V. R., \& Mulakaluri, R. R.
(2014). Candidal carriage, isolation and species variation in patients undergoing radiotherapy and chemotherapy for head and neck tumours. Journal of Dr. NTR University of Health Sciences, 3, 28-34.

Brunner, E., \& Langer, F. (2000). Nonparametric analysis of ordered categorical data in designs with longitudinal observations and small sample sizes. Biometrical Journal, 42, 663-675.

Eliasson, L., Carlén, A., Almståhl, A., Wikström, M., \& Lingström, P. (2006). Dental plaque $\mathrm{pH}$ and microorganisms during hyposalivation. Journal of Dental Research, 85, 334-338.

Ericsson, Y. (1959). Clinical investigations of the salivary buffering action. Acta Odontologica Scandinavica, 17, 131-165.

Freitas, E. M., Nobre, S. A. M., Pires, M. B. O., Faria, R. V. J., Batista, A. U. D., \& Bonan, P. R. F. (2013). Oral Candida species in head and neck cancer patients treated by radiotherapy. Auris, Nasus, Larynx, 4, 400-404.

Gaetti-Jardim, E., Jr., Avila-Campos, M. J., Ciesielski, F. I. N., \& Sousa, F. R. N. (2011). Occurrence of yeasts, pseudomonads and enteric bacteria in the oral cavity of patients undergoing head and neck radiotherapy. Brazilian Journal of Microbiology, 42, 1047-1055.

Gaetti-Jardim, E., Jr., Monti, L. M., Ciesielski, F. I. N., Gaetti-Jardim, E. C., Okamoto, A. C., Schweitzer, C. M., et al. (2012). Subgingival microbiota from Cebus apella (capuchin monkey) with different periodontal conditions. Anaerobe, 18, 263-269.

Gonçalves, M. O., Coutinho-Filho, W. P., Pimenta, F. P., Pereira, G. A., Pereira, J. A. A. Mattos-Guaraldi, A. L., et al. (2007). Periodontal disease as reservoir for multi-resistant and hydrolytic enterobacterial species. Letters in Applied Microbiology, 44, 488-494.

Jham, B. C., França, E. C., Oliveira, R. R., Santos, V. R., Kowalski, L. P., \& Freire, A. R. S. (2007). Candida oral colonization and infection in Brazilian patients undergoing head and neck radiotherapy: A pilot study. Oral Surgery Oral Medicine Oral Pathology Oral Radiology and Endodontics, 103, 355-358.

Leung, W. K., Jin, L. J., Yam, W. C., \& Samaranayake, L. P. (2001). Oral colonization of aerobic and facultative anaerobic Gram-negative rods and cocci in irradiated, dentate: Xerostomic individuals. Oral Microbiology and Immunology, 16, 1-9.

Meng, L., Liu, J., Peng, B., Fan, M., Nie, M., Chen, Z., et al. (2005). The persistence of $S$. mutans in nasopharyngeal carcinoma patients after radiotherapy. Caries Research, 39, 484-489.

Minhas, S., Kashif, M., Altaf, W., \& Nagi1, A. H. (2016). Oral candidiasis: Complication of concomitant chemo-radiotherapy in patients with oral squamous cell carcinoma. British Microbiology Research Journal, 11, 1-11.

Mizutani, S., Ekuni, D., Tomofuji, T., Azuma, T., Kataoka, K., Yamane, M., et al. (2015). Relationship between xerostomia and gingival condition in young adults. Journal of Periodontal Research, 50, 74-79.

Scarantino, C., Leveque, F., Swann, S., White, R., Schulsinger, A., Hodson, I., et al. (2006) Effect of pilocarpine during radiation therapy: Results of RTOG 97-09, a phase III randomized study in head and neck cancer patients. Journal of Supportive Oncology, 4, 252-258.

Schuurhuis, J. M., Stokman, M. A., Witjes, M. J. H., Langendijk, J. A., Van Winkelhoff, A. J., Vissink, A., et al. (2016). Head and neck intensity modulated radiation therapy leads to an increase of opportunistic oral pathogens. Oral Oncology, 58, 32-40.

Sonalika, W. G., Tayaar, S. A., Bhat, K. G., Patil, B. R., \& Muddapur, M. V. (2012). Oral microbial carriage in oral squamous cell carcinoma patients at the time of diagnosis and during radiotherapy. A comparative study. Oral Oncology, 48, 881-886.

Sun, H. B., Gao, X. J., Deng, J., Li, N. L., \& Lu, H. J. (2010). Progress of oral sequelae during head-neck radiotherapy. Chinese Journal of Dental Research, 13, 51-55.

Vanhoecke, B., De Ryck, T., Stringer, A., Van de Wiele, T., \& Keefe, D. (2015). Microbiota and their role in the pathogenesis of oral mucositis. Oral Diseases, 21, 17-30.

Yogitha, P. V. V., Lakshmi, N., Lakshmi, K. R., Krishna, P. B. M., \& Cheemala, S. S. (2015). Isolation and speciation of genus Candida in patients undergoing chemotherapy and radiotherapy for head and neck tumours. International Journal of Research in Medical Science, 3, 1189-1194. 